\title{
AVALIAÇÃO DE ANTÍGENOS DE CYSTICERCUS CELLULOSAE NO IMUNODIAGNÓSTICO DA CISTICERCOSE HUMANA PELA HEMAGLUTINAÇÄO INDIRETA
}

\author{
E. NASCIMENTO (1) e W. MAYRINK (1)
}

\begin{abstract}
RESUMO
Os Autores avaliaram os antígenos de Cysticercus cellulosae para o imunodiagnóstico da cisticercose humana pela reação de hemaglutinação indireta. Observaram que os antígenos de escólex (ES) e ES pico II são mais específicos que os antígenos de C. cellulosae total (CC), líquido vesicular e de membrana (ME). Usando soros de pacientes com cisticercose comprovada parasitologicamente, obtiveram uma sensibilidade de 0,80 para o ES, 0,84 para o ES II e especificidade 1,0, quando os soros foram usados na diluição 1:8 para 0 antígeno ES e 1:16 para 0 antígeno ES II.
\end{abstract}

\section{N T R O D U C $\tilde{A} O$}

No imunodiagnóstico da cisticercose humana são conhecidas as dificuldades para se detectar anticorpos anti-cisticercos; a pesquisa de anticorpos no líquido cefalorraqueano (LCR) é de grande utilidade no diagnóstico da neuro. cisticercose, entretanto a mesma pesquisa no soro seria facilitada devido a sua fácil obtenção em relação ao LCR que é obtido através da punção medular.

Empregando extrato total de Cysticercus cellulosae e a reação de hemaglutinação indireta (HI) para detectar anticorpos circulantes anti-cisticercos, BIAGI \& col. ${ }^{1}$, encontraram uma positividade de $92 \%$ em 13 soros de pacientes com cisticercose comprovada. PROCTOR \& col. "1, utilizando a mesma técnica e antígeno de $\mathbf{C}$. cellulosae extraído em solução salina, encontraram $85 \%$ de positividade em 48 soros de pacientes com cisticercose comprovada e $17 \%$ em soros de pacientes com teníase por Taenia solium ou T. saginata.

POWELL \& col. 10 usando como antígeno 0 extrato total de C. cellulosae e a HI para 0 diagnóstico da cisticercose, concluíram ser esse método de grande valor na detecção de an ticorpos anti-cisticercos na forma assintomática e na epilepsia associada a cisticercose.

Estudos recentes sobre o relacionamento entre antígenos do $\mathbf{C}$. cellulosae e da $\mathbf{T}$. solium, através de imune soros, demonstraram a exis tência de componentes comuns entre os antígenos do $\mathbf{C}$. cellulosae e da $\mathbf{T}$. solium (NASCI. MENTO \& ARAUUJO ${ }^{8}$ ).

O presente trabalho tem como objetivo avaliar a especificidade e a sensibilidade dos antígenos de $\mathbf{C}$. cellulosae para o imunodiag. nóstico da cisticercose humana pela hemaglu. tinação inđireta.

\section{MATERIAL E MÉTODOS}

Antígenos - Os Cysticercus cellulosae (CC) foram obtidos do coração, língua e músculos esqueléticos de suínos naturalmente infectados. Após serem retirados dos músculos, os cisticercos foram lavados em banhos sucessi-

(1) Trabalho realizado com apoio do Conselho Nacional de Desenvolvimento Científico e Tecnológico (CNPq) e da Fi nanciadora de Estudos e Projetos (FINEP), no Departamento de Parasitologia, Instituto de Ciências Biológicas da Universidade Fecleral de Minas Gerais, Caixa Postal 2486, 30.000 - Belo Horizonte, Minas Gerais, Brasil 
NASCIMENTO, E. \& MAYRINK, W. - Avaliação de antígenos de Cysticercus cellulosae no imunođiagnostico da cisticercose humana pela hemaglutinaçāo indireta. Rev. Inst. Med. trop. São Paulo 26:289-294, 1984.

vos de água destilada e de solução salina tamponada com fosfato (SST) $0,15 \mathrm{M} \mathrm{pH} \mathrm{7,2.} \mathrm{Par-}$ te dos cisticercos integros foi liofilizada e parte foi separada em membrana (MF) e escólex (ES) com auxílio de bisturi e microscópio esterosicópico.

$\mathrm{O}$ antígeno de líquido vesicular (LI) foi colhido diretamente das vesículas com auxilio de uma seringa adaptada com agulha $15 \times 10$. As partes do $\mathbf{C}$. cellulosae: ME, ES, LI e o CC total foram liofilizadas, sendo a extração dos antígenos realizada segundo método descrito por NASCIMENTO \& ARAÚJO ${ }^{7}$.

A padronização da concentração protéica đos antígenos foi feita de acordo com o método descrito por LOWRY \& col. 6 .

Soros dos pacientes - Os soros dos pacientes com neurocisticercose comprovada por cirurgia foram obtidos no Departamento de Neurologia do Hospital das Clínicas da Universidade Federal de Minas Gerais, enquanto que os demais soros de pacientes com teniase (T. solium ou T. saginata), ancilostomose, esquistossomose, ascaridiase, tricocefalose, estrongiloidose e doença de Chagas foram obtidos no Departamento de Parasitologia do Instituto de Ciências Biológicas da Universidade Federal de Minas Gerais. Como padrőes negativos foram usados soros de recém-nascidos Norte-Americanos, obtidos na divisão de alergia, imunologia e doenças infecciosas da Palo Alto Medical Research Foundation, Palo Alto, Califórnia, USA. Todos os soros foram diluídos inicialmente a $1: 4$ em SSL.

Fracionamento de antígenos - As frações dos antígenos ES e LI usadas no presente trabalho foram obtidas como descrita por NASCIMENTO \& ARAƯJO 8 .

Hemaglutinação indireta - Foi executada usando-se placas tipo $\mathrm{V}$, segundo NASCIMENTO \& ARAÛJO ${ }^{8}$. Os eritrócitos humanos, O Rh negativos, foram fixados pelo formaldeído segundo BUTLER ${ }^{3}$, HOSHINO-SHIMIZU \& col.5. $\mathrm{Na}$ sensibilização dos eritrócitos foram usados $3 \mathrm{ml}$ de uma suspensão de células a $10 \%$, para cada antígeno. Inicialmente, as células foram lavadas por centrifugação a $1000 \mathrm{~g}$, cinco vezes com $10 \mathrm{ml}$ cada vez de solução de $\mathrm{NaCl}$ a $0,85 \%$, durante três minutos. $O$ sedimento de eritrócitos foi ressuspenso em $10 \mathrm{ml}$ de PBS pH 7,2 contendo áciđo tânico a 1:15000 e incu- bado em banho-maria a $37^{\circ} \mathrm{C}$, durante $30 \mathrm{minu}$ tos. Em seguida as células foram lavadas por centrifugação, cinco vezes em soluçāo salina. $O$ sedimento foi ressuspenso em $10 \mathrm{ml}$ de PBS pH 6,4 , contendo um antígeno de cada na concentração de 150 microgramas de proteínas por mililitro e incubação por 30 minutos a $37^{\circ} \mathrm{C}$. Após a incubação, os eritrócitos foram novamente lavados cinco vezes em solução salina contendo $1 \%$ de soro normal, inativado, de coelho. Finalmente os eritrócitos foram ressus pensos a $1 \%$ em solução estabilizadora preparada segundo HOSHINO-SHIMIZU \& col. 5 e conservados a $4^{\circ} \mathrm{C}$. Vinte e cinco microlitros do antígeno foram gotejados em cada orifício da placa contendo 50 microlitros da diluição dos soros e a placa em seguida agitada por 30 segundos em vibrador (Mixtron, Toptronix, Brasil). A leitura foi realizada após duas horas à temperatura ambiente.

Sensibilidade e especificidade - Os cálculos desses índices foram feitos de acordo com BUCK \& GART ${ }^{2}$.

\section{RESULTADOS}

Anticorpos anti-cisticercos foram detecta dos pelos antígenos CC, ES, ES II, LI, LI II e ME até nas diluiçōes de soros de 1:512 ou 1:1024, de pacientes com cisticercose comprovada (Tabela I). Foram observadas reações cruzadas com teníase por $\mathbf{T}$. solium ou $\mathbf{T}$. saginata nas diluiçōes de soro de até $1: 32 \mathrm{com}$ antígeno CC, 1:8 com antígeno ES, 1:64 com antígeno ES II, 1:16 com antigeno LI e 1:8 com antígeno ME (Tabela I). Para pacientes com ascaridíase, tricocefalose, esquistossomose e sífilis foram observadas reações cruzadas com soros puros ou diluidos até $1: 8$, com os antígenos CC, ES II e LI II.

As diluições dos soros nos quais as reações de hemaglutinação mostrou especificidade máxima (índice 1,0) variaram segundo os diferentes antígenos e corresponderam a diferentes índices de sensibilidade. Estes dados estão reunidos na Tabela II.

\section{DISCUSSĀO}

Em geral a reação de hemaglutinação indi reta para cisticercose é considerada como a técnica mais sensível do que algumas reações de precipitação e mesmo em relação à fixação 
NASCIMENTO, E. \& MAYRINK, W. - Avaliação de antígenos de Cysticercus cellulosae no imunodiagnóstico da cisticercose humana pela hemaglutinação inđireta. Rev. Inst. Med. trop. São Paulo 26:289-294, 1984.

T A B E L A I

Avaliação comparativa de antígenos de Cysticercus cellulosae e soros de pacientes com cisticercose, teníases e outras parasitoses

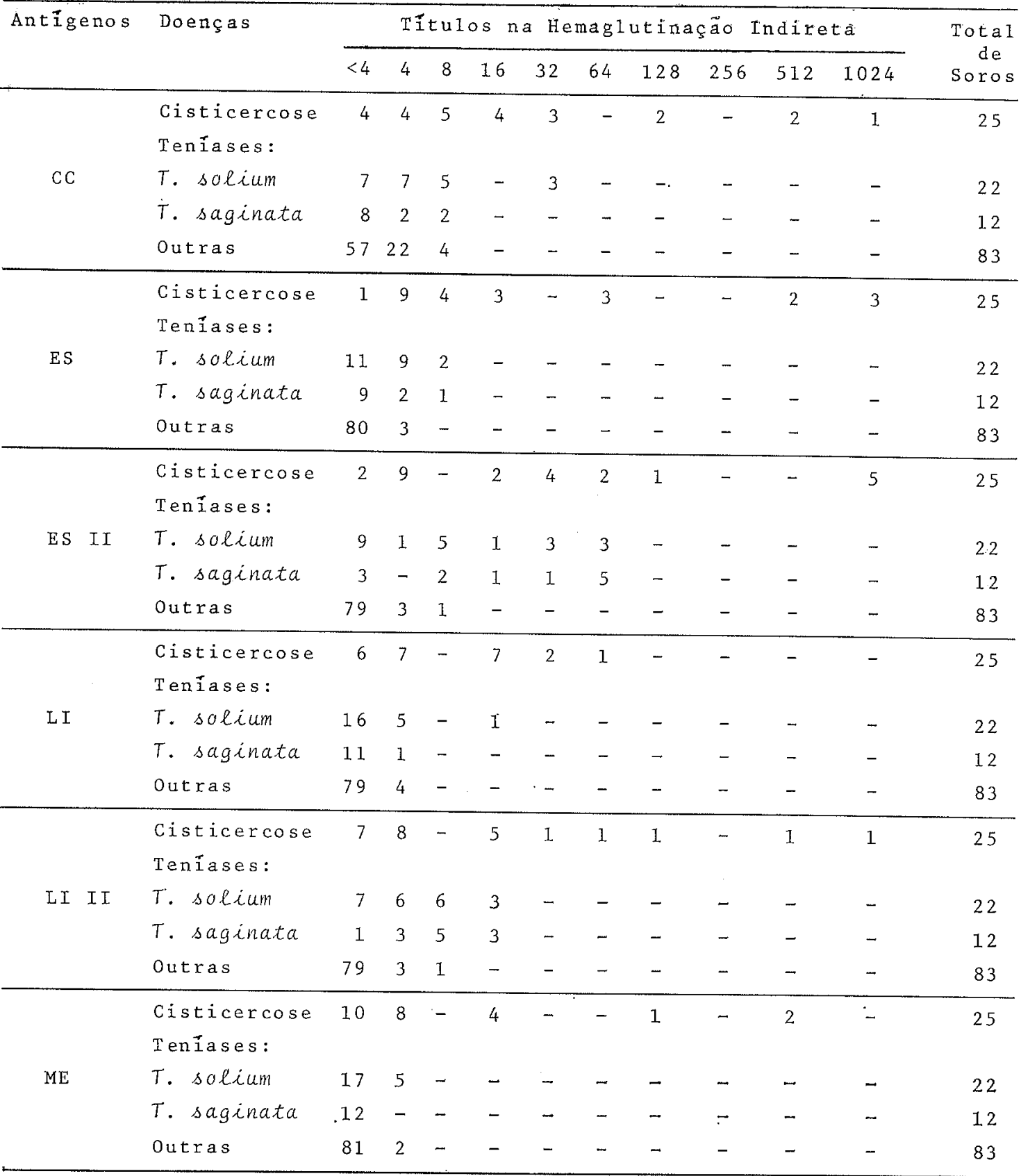

Soros de pacientes com outras parasitoses (83): Doença de Chagas (10), Esquistossomose (7), Tricocefalose (7), Ancilostomose (4), Estrongiloidose (2), Ascaridíase (6) e soros padröes negativos (22)

de complemento (BIAGI \& col.1; PROCTOR \& col. 11). No entanto, através dessa técnica ainda são observados resultados falso-positivos, provavelmente devido ao uso de antígenos não padronizados.
Reações cruzadas com o antígeno CC total foram observadas em soros de pacientes portadores de teníase, sífilis e outras parasitoses (Tabela I). Isto também aconteceu com os demais antígenos, mas em menor proporção. Tais 


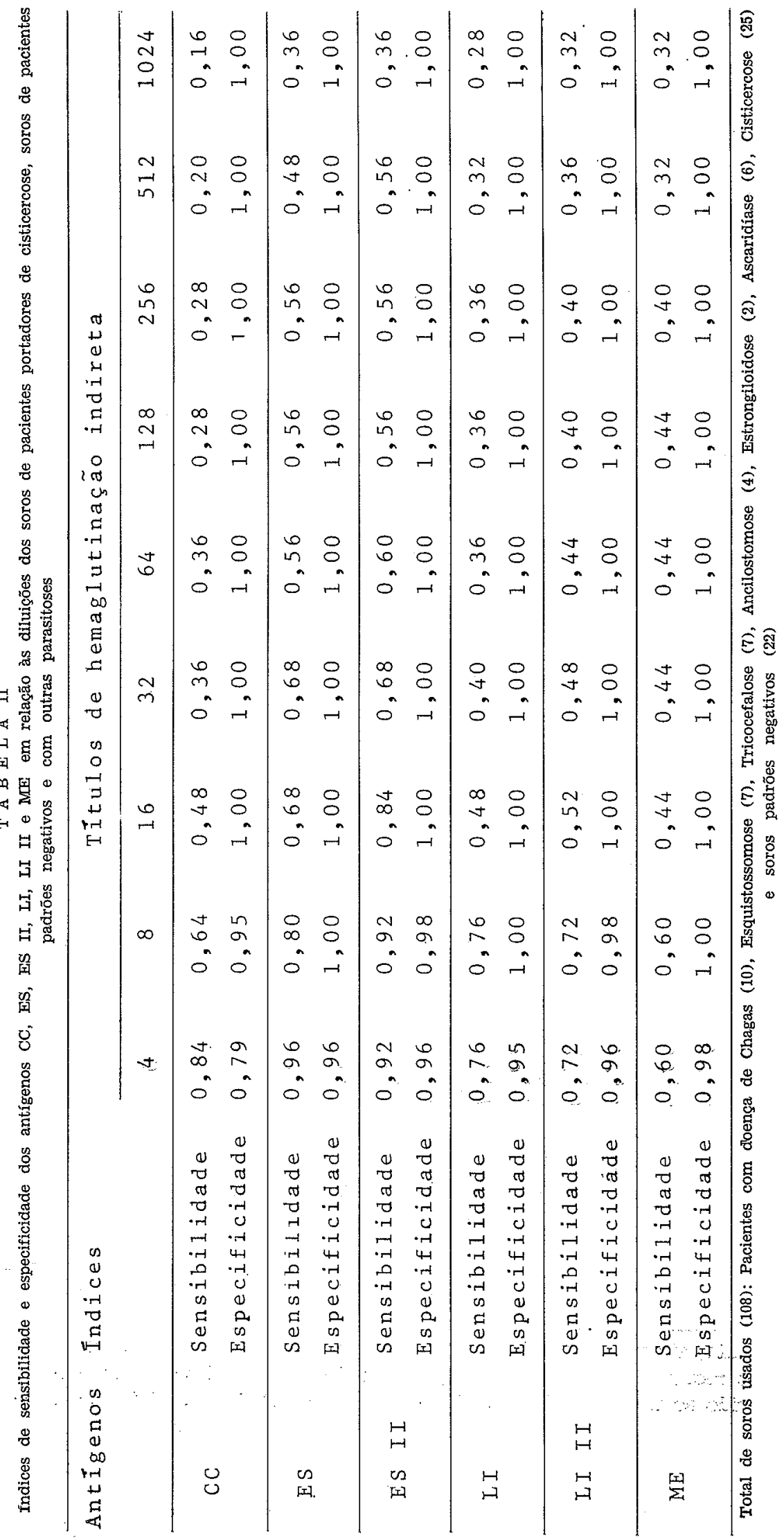


NASCIMENTO, E. \& MAYRINK, W. - Avaliação de antígenos de Cysticercus cellulosae no imunodiagnóstico da cisticercose humana pela hemaglutinação indireta. Rev. Inst. Med. trop. São Paulo 26:289-294, 1984.

dados confirmam os resultados obtidos por NASCIMENTO \& ARAÚJO's usando imune soros frente a antígenos de C. cellulosae e T. solium. Reações cruzadas, sempre com títulos menores do que 1:8, também, foram observadas em soros de pacientes com sífilis, ascari díase, tricocefalose e esquistossomose mansô nica. Nas diluições de soros em que não mais se observaram tais reações cruzadas, os antígenos CC, LI, LI II e ME apresentaram indices de sensibilidade $0,48,0,76,0,52,0,60$ (Tabela II).

Diluições discriminantes a 1:8 dos soros para o antígeno ES e 1:16 para o ES II devem ser usadas e não a 1:64 como preconizada por NAHAJAN \& col. ${ }^{9}$, porque julgamos que diluições abaixo de 1:8 diminuem a especificidade $\mathrm{e}$ acima desse título podem levar a resultados falso-negativos com os nossos antígenos; possivelmente, devido a presença no soro de pequena concentração de anticorpos circulantes anti-cisticerco ou que a maior reatividade imunológica dos pacientes esteja relacionada com a. localização, viabilidade e ou com o número de cisticercos no organismo. Tal hipótese foi tam. bém proposta por HERBERT \& OBERG ${ }^{4}$, na cisticercose suina.

Com o extrato total de C. cellulosae foi obtida uma sensibilidade de 0,84 e uma especificidade de $0,79 \mathrm{em} 25$ soros de pacientes com cisticercose, diluídos a 1:4 (Tabela II). BIAGI \& col. ${ }^{1}$ observaram uma positividade de $92 \%$ em 13 soros testados e PROCTOR \& col. 11 encontraram anticorpos em $85 \%$ dos 48 soros testados.

Os antígenos ES e ES II foram os mais específicos para detectar anticorpos anti-cisticercos. Para uma especificidade de 1,0 foi observada sensibilidade $0,80 \mathrm{com}$ antígenos ES, na diluição 1:8 dos soros e $0,84 \mathrm{com}$ antígeno ES II, na diluição 1:16 dos soros. Usando o antígeno ES e soros diluídos a 1:8 foi observađo menor número de reações cruzadas com teníase, do que para o antígeno ES II, mesmo usando soros diluídos a 1:16. Tal fato, possivelmente seja devido a existência de maior concentração de componentes antigênicos comuns entre o ES II e as tênias. Pelos resultados obtidos estamos convencidos de que os antígenos ES e ES II deverão ser usados na detecção de anticorpos no soro de pacientes com cisticercose pela hemaglutinação indireta.

\section{SUMMARY}

Evaluation of antigens from Cysticercus cellulosae for immunodiagnosis of human cysticercosis by haemagglutination test

The Authors evaluated the use of antigens from Cysticercus cellulosae in the immunodiagnosis of human cysticercosis by haemagglutination test. The extract prepared from the escolex and its chromatographic peak II were more specific than those from whole larvae, vesicular liquid and membrane antigens. Using sera from patients with parasitologically proven cysticercosis, positive reactions were obtained with a sensitivity index of 0.80 for escolex antigen and 0.84 escolex peak II antigen. Specificity of 1.0 was obtained for sera diluted at 1:8 for ES and 1:16 for ES II antigens.

\section{REFERENGIAS BIBLIOGRAFICAS}

1. BIAGI, F. F.; NAVARRETE, F.; PINA, A.; SANTTAGO, A. M. \& TAPIA, L. - Estudio de tres reaciones sorologicas en el diagnostico de la cisticercosis. Rev. Med. Hosp. General Mexico II: 501-508, 1961.

2. BUCK, A. A. \& GART, J. J. - Comparison of a screening test and a reference test in epidemiologic studies. I. Indices of agreement and their relation to prevalence. Am. J. Epidemiol. 83: 586-592, 1965.

3. BUTLER, W. T. - Hemagglutination studies with formalinized erythocytes. Effect of bis-diazo-benzidine and tannic acid treatment on sensibilization by soluble antigen. J. Immunol. 90: 663-671, 1963.

4. HERBERT, I. V. \& OBERG, C. - Immunology of larval $\mathbf{x}$. solium infections. Parasitic Zoonoses. Clinical and Experimental Studies. Edited by Soulsby. New York, Academic Press, 1974, 199-211.

5. HOSHINO-SHIMIZU, S.; CAMARGO, M. \& NAGASSE, T. K. - Stable polysaccharide-hemagglutination reagent for the diagnosis of acute or recent Trypanosoma cruzi infection. Rev. Inst. Med. trop. São Paulo 20: 208-212, 1978.

6. LOWRY, O. H. ; ROSEBROUCH, N. J.; FARR, L. \& RANDALL, R. J. - Protein measurement with the folin phenol reagent. J. Biol. Chem. 193: 265-275, 1951.

7. NASCIMENTO, E. \& ARAƯJO, F. G. - Estudos imunoquímicos em extratos aquosos de larvas e adultos de Taenia solium. I. Imunogenicidade e composição antigênica. Rev. Inst. Med. trop. São Paulo 24: 353 358,1982

8. NASCIMENTO, E. \& ARAŨJO, F. G. - Estudos imunoquímicos em extratos aquosos de Taenia solium. II. Relacionamento antigênicos, pađröes cromatografi- 
cos, atividade imunológica do escólex. Rev. Inst. Med. trop. São Paulo 24: 359-363, 1982.

9. NAHAJAN, R. C.; CHITKARA, N. L. \& CHOPRA, J. S. - Evaluation of cysticercosis and adult worm antigen in serodiagnosis of cysticercosis. Indian I. Med. Res. 62: 1310, 1974.

10. POWELL, S. J.; PROCTOR, E. M.; WILMOT, A. J. \& BARNETT, A. M. - Neurological complications of cysticercosis in Africans; a clinical and sorological in Africa. Ann, trop. Med. Parasitol. 60: 146-151, 1966.

11. PROCTOR, E. M.; POWELL, S. J. \& ELSDSON-DEW, E. - The sorological diagnosis of cysticercosis in Africa. Ann. trop. Med. Parasitol. 60: 146-151, 1966.

Recebido para publicaçāo em 29/8/1983. 